\title{
miRFA: an automated pipeline for microRNA functional analysis with correlation support from TCGA and TCPA expression data in pancreatic cancer
}

\author{
Emmy Borgmästars $^{1 *}$ D, Hendrik Arnold de Weerd ${ }^{2,3}$, Zelmina Lubovac-Pilav ${ }^{2}$ and Malin Sund ${ }^{1}$
}

\begin{abstract}
Background: MicroRNAs (miRNAs) are small RNAs that regulate gene expression at a post-transcriptional level and are emerging as potentially important biomarkers for various disease states, including pancreatic cancer. In silicobased functional analysis of miRNAs usually consists of miRNA target prediction and functional enrichment analysis of miRNA targets. Since miRNA target prediction methods generate a large number of false positive target genes, further validation to narrow down interesting candidate miRNA targets is needed. One commonly used method correlates miRNA and mRNA expression to assess the regulatory effect of a particular miRNA.

The aim of this study was to build a bioinformatics pipeline in R for miRNA functional analysis including correlation analyses between miRNA expression levels and its targets on mRNA and protein expression levels available from the cancer genome atlas (TCGA) and the cancer proteome atlas (TCPA). TCGA-derived expression data of specific mature miRNA isoforms from pancreatic cancer tissue was used.

Results: Fifteen circulating miRNAs with significantly altered expression levels detected in pancreatic cancer patients were queried separately in the pipeline. The pipeline generated predicted miRNA target genes, enriched gene ontology (GO) terms and Kyoto encyclopedia of genes and genomes (KEGG) pathways. Predicted miRNA targets were evaluated by correlation analyses between each miRNA and its predicted targets. MiRNA functional analysis in combination with Kaplan-Meier survival analysis suggest that hsa-miR-885-5p could act as a tumor suppressor and should be validated as a potential prognostic biomarker in pancreatic cancer.
\end{abstract}

Conclusions: Our miRNA functional analysis (miRFA) pipeline can serve as a valuable tool in biomarker discovery involving mature miRNAs associated with pancreatic cancer and could be developed to cover additional cancer types. Results for all mature miRNAs in TCGA pancreatic adenocarcinoma dataset can be studied and downloaded through a shiny web application at https://emmbor.shinyapps.io/mirfa/.

Keywords: miRNA functional analysis, miRNA target prediction, Functional enrichment, Mature miRNA, TCGA, TCPA, Pancreatic cancer

\footnotetext{
* Correspondence: emmy.borgmastars@umu.se

'Department of Surgical and Perioperative Sciences, Umeå University, Umeå,

Sweden

Full list of author information is available at the end of the article
}

(c) The Author(s). 2019 Open Access This article is distributed under the terms of the Creative Commons Attribution 4.0 International License (http://creativecommons.org/licenses/by/4.0/), which permits unrestricted use, distribution, and reproduction in any medium, provided you give appropriate credit to the original author(s) and the source, provide a link to the Creative Commons license, and indicate if changes were made. The Creative Commons Public Domain Dedication waiver (http://creativecommons.org/publicdomain/zero/1.0/) applies to the data made available in this article, unless otherwise stated. 


\section{Background}

MicroRNAs (miRNAs) are small RNAs of about 19-24 nucleotides [1]. Two miRNA isoforms, termed $-3 p$ and $-5 p$ arms, are formed from stem-loops that originate from miRNA genes. Usually one of the mature miRNAs, called the passenger strand, is degraded and the other strand, often referred to as guide strand, is playing a role in miRNA-mediated regulation [1]. Nonetheless, both strands may act in miRNA-mediated regulation. MiRNAs are generally considered down-regulators of mRNAs at a post-transcriptional level, but they can also act as upregulators [2, 3]. In miRNA-mediated down-regulation, translational repression is usually the primary event followed by mRNA degradation [4]. MiRNA-mediated upregulation may occur indirectly by interfering with repressive miRNA ribonucleoprotein complex (miRNPs) or directly by the activity of miRNPs [5]. Positive regulation seems to be restricted to certain cell conditions, for instance cells in G0 cell cycle state [2].

Pancreatic ductal adenocarcinoma (PDAC) is the most common form of malignant pancreatic neoplasms [6], often diagnosed at a late clinical stage, with very poor prognosis due to early metastatic spread [7]. The most commonly used diagnostic biomarker today is carbohydrate antigen 19-9 (CA 19-9). However, this biomarker has several disadvantages including suboptimal specificity, with elevated levels detected in other diseases, and false negative detections [8]. Hence, research efforts need to be directed towards finding novel, more reliable biomarkers. MiRNAs are highly stable in blood and have been studied as potential non-invasive biomarkers in numerous diseases, including pancreatic cancer $[7,9,10]$. Recently, 15 circulating miRNAs with significantly altered expression levels at PDAC diagnosis were identified and a combination of these miRNA biomarkers was shown to outperform CA 19-9 as a diagnostic marker in terms of area under curve (AUC) [7].

In order to understand the role of miRNA biomarkers, in silico-based functional analysis can be performed, which typically consists of target prediction following functional enrichment analysis of identified miRNA targets [11]. Several $R$ packages and web resources exist for miRNA functional analysis. MultiMiR [12] and RBiomirGS [13] are $R$ packages that perform miRNA target prediction, while RBiomirGS performs functional enrichment analysis as well. The $\mathrm{R}$ package MiRComb utilizes miRNA-mRNA expression correlations followed by miRNA target prediction based on negatively correlated targets [14]. MiRLAB performs target prediction and enrichment analysis in combination with mRNA and miRNA expression data provided by the user or from the cancer genome atlas (TCGA) to infer regulatory relationships [15]. Recently, a shiny web application named miRCancerdb was published, enabling users to study correlations between miRNA expression to its targets or non-targets on mRNA and protein expression levels using TCGA data [16, 17]. Another example of a web-based tool is DNA intelligent analysis (DIANA)mirPath v3.0 [18], which performs miRNA target prediction and functional enrichment generating a list of target genes as well as gene ontology (GO) terms and Kyoto encyclopedia of genes and genomes (KEGG) pathways.

MiRNA target predictions usually generate a high false-positive rate and the most preferable way of evaluating miRNA target predictions is experimental validation [19]. This is however not always possible due to a high number of predicted targets, although databases for collected experimentally validated miRNA targets exist [20]. Validation of identified miRNA targets is a challenge and an intermediate step from prediction to wet lab validation is of great benefit to narrow down interesting candidates. One in silico-based validation approach is to correlate miRNA and mRNA expression levels in combination with miRNA target prediction. A common approach when analyzing the regulatory effect of specific miRNAs is to study changes on mRNA level, whereas regulatory effect of miRNA might in some cases only impact the protein level [4]. In a correlation analysis approach, it is helpful to include protein expression levels since mRNA levels do not always correlate with protein expression levels [21]. Another limitation of some studies is the assumption that miRNAs act as down-regulators of target genes, which is why mainly negative correlation is often considered [22, 23]. As mentioned, positive miRNA-mediated regulation may also occur [2,3] and hence it is important to also include positive correlations.

Here, we describe miRNA functional analysis (miRFA), a pipeline built in $\mathrm{R}$ that provides following features:

1) MiRNA target prediction using two target prediction databases and one experimentally validated target database

2) Correlation analysis between miRNA and its predicted target genes on mRNA and protein expression levels derived from TCGA pancreatic adenocarcinoma (PAAD) project

3) Functional enrichment of significantly correlated miRNA targets

The novelty of our pipeline is the combination of including mature miRNA expression levels (isoform quantification) from TCGA-PAAD, protein expression levels from the cancer proteome atlas (TCPA) [24], and functional enrichment of both negatively and positively correlated miRNA-targets. Combination of the abovementioned features in one tool may facilitate the research in miRNA biomarker discovery in pancreatic 
cancer. The tool was built in $\mathrm{R}$ and to make it even more accessible to users not familiar with $\mathrm{R}$, we developed a shiny web app available at https://emmbor.shinyapps.io/mirfa/, where results for all miRNAs detected in TCGA-PAAD can be retrieved [17].

\section{Results}

An overview of the miRFA pipeline is shown (Fig. 1). The input is a mature miRNA name and the output contains lists of miRNA target genes, Venn diagrams of target genes, miRNA targets correlations on mRNA and protein expression levels, and significantly enriched GO terms and KEGG pathways. For correlation analysis, we implemented miRNA isoform quantification data from TCGA in order to separate between expression levels of $-3 p$ and $-5 p$ arms of mature miRNAs. To illustrate the difference between expression levels of the precursor miRNA gene and the mature miRNA isoforms, hsa-mir-144 was plotted as an example together with expression levels of mature isoforms hsa-miR-144-3p and hsa-miR-144-5p (Fig. 2).
The expression levels of the precursor hairpin hsa-mir144 is more similar to the mature miRNA hsa-miR-144$5 p$ compared to hsa-miR-144-3p.

\section{Predicted miRNA targets partially overlap} MiRNA target prediction was performed in three databases; DIANA-TarBase v7 [25], DIANA-microT-CDS [26] and TargetScan v7.1 [27]. The largest number of predicted targets was generally identified from TargetScan, exceeding 3000 predicted target genes for many of the miRNAs (Fig. 3). That said, no target gene was found in TargetScan for hsa-miR-101-3p.

A moderately sensitive threshold of 0.7 was used for DIANA-microT-CDS which affects the number of predicted miRNA targets. Defining a less restrictive threshold could generate more targets that are also present in DIANA-TarBase, but it could also introduce a higher number of false positives. The generated Venn diagrams show that some of the miRNA targets in DIANA-TarBase were not identified by the in silico prediction tools (Additional

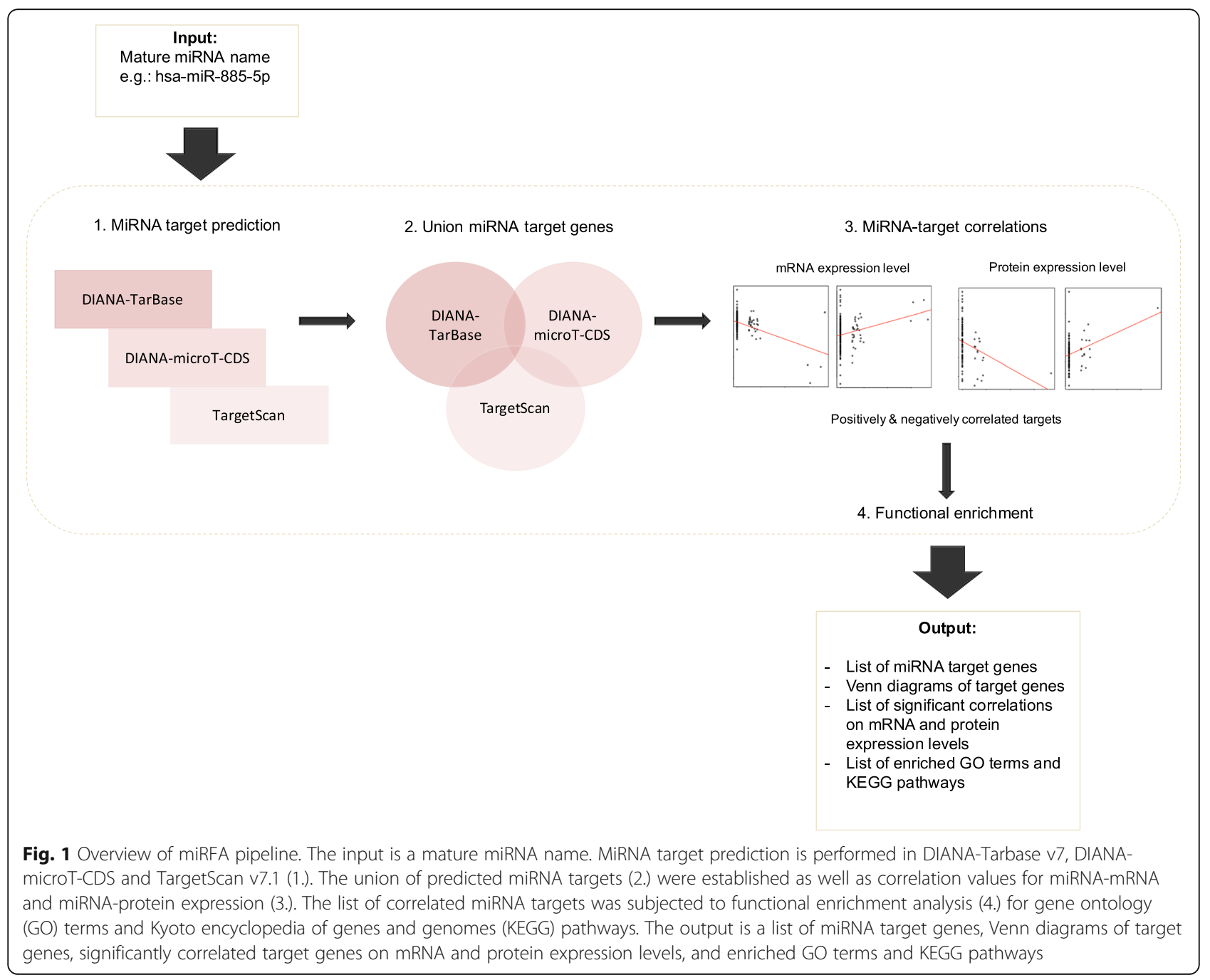




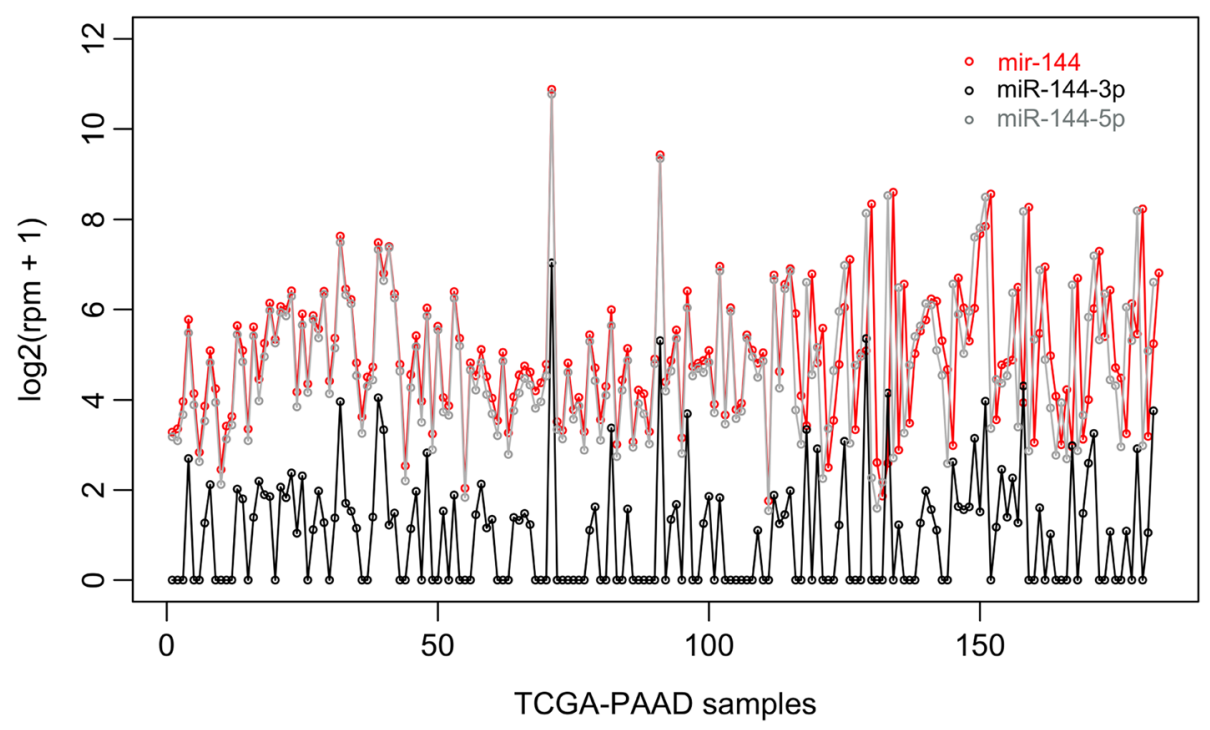

Fig. 2 The difference between hsa-mir-144, hsa-miR-144-3p and hsa-miR-144-5p. Expression values were plotted for 183 TCGA-PAAD samples. Hsa-mir-144 (mir-144) represents the precursor hairpin expression, whereas hsa-miR-144-3p (miR-144-3p) and hsa-miR-144-5p (miR-144-5p) represents the mature miRNA isoforms expression. Rpm = reads per million counts, TCGA = the cancer genome atlas, PAAD $=$ pancreatic adenocarcinoma

file 6: Figure S1). The opposite scenario also occurs, that targets predicted by TargetScan or DIANA-microT-CDS have not been experimentally validated.

\section{MiRNA-mRNA correlations}

As miRNA target prediction tools can render many false positives, in silico evaluation data is useful to narrow down interesting gene candidates. To identify target genes that may have a role in pancreatic cancer progression, expression data of miRNAs, mRNAs, and proteins from pancreatic cancer tissue was used to analyze correlations between the query miRNA and its corresponding target genes on mRNA and protein levels.

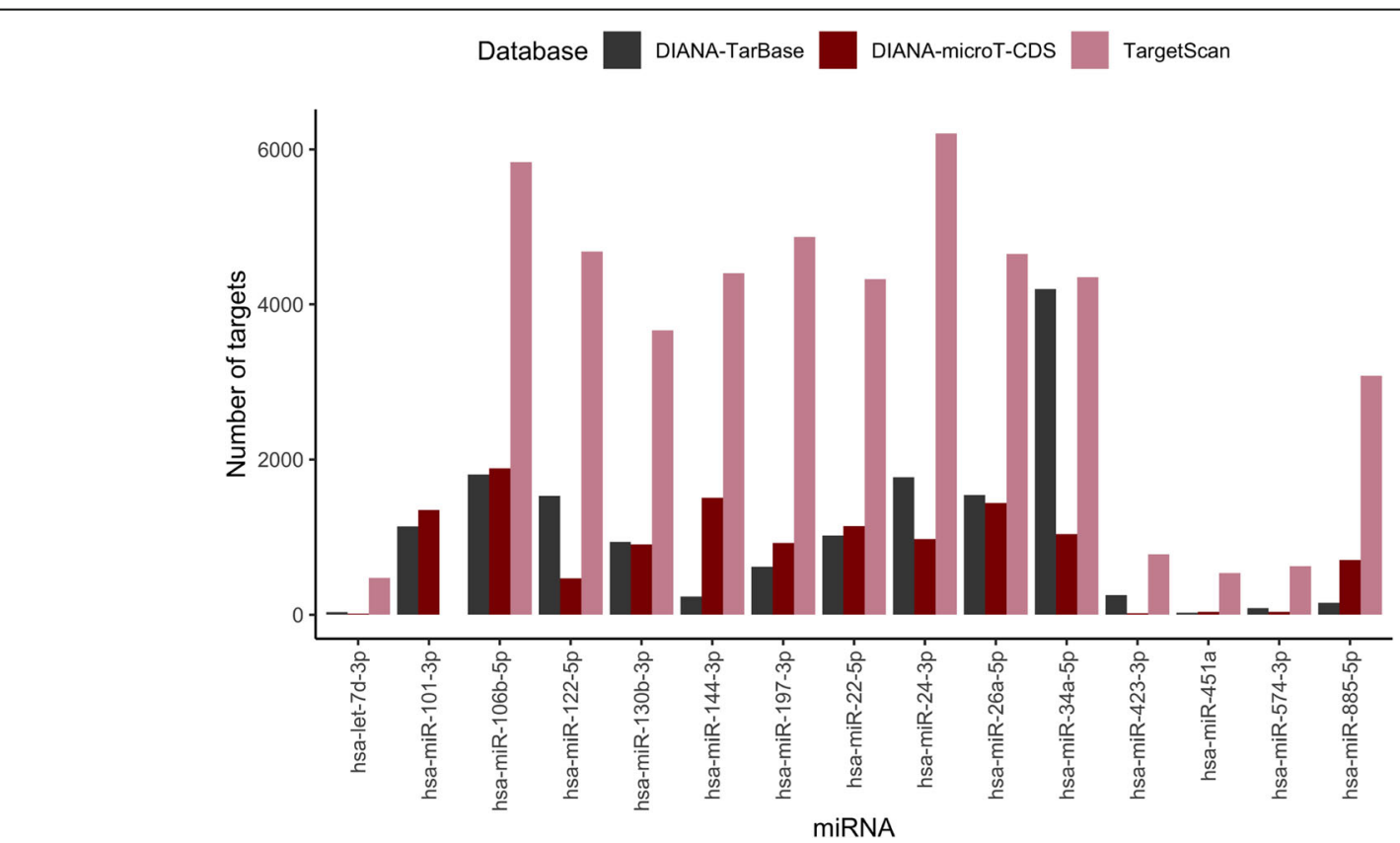

Fig. 3 Number of predicted miRNA targets by DIANA-TarBase v7, DIANA-microT-CDS and TargetScan v7.1 for 15 miRNAs. The $x$ axis shows every miRNA queried and the $y$ axis shows the number of predicted miRNA targets 
In general, the number of significant correlations was low compared to the number of predicted targets (Fig. 4). For all 15 miRNAs combined, a total of 10,754 significant correlations (adjusted $p$-value $<0.05$ ) were found, of which 4203 were positively correlated (Pearson's correlation coefficient; $\mathrm{PCC}>0$ ), and 6551 negatively correlated $(\mathrm{PCC}<0)$. Hsa-miR-106b-5p obtained the highest number of negative correlations and hsa-miR-24-3p the highest number of positive correlations.

\section{MiRNA-protein correlation}

Correlation analysis of miRNA-protein expression levels was performed on 98 TCGA-PAAD samples. In total, 43 significant correlations (adjusted $p$-value $<0.05$ ) were identified on protein level, consisting of 22 negatively correlated $(\mathrm{PCC}<0)$ and 21 positively correlated $(\mathrm{PCC}>$ 0 ). Only five miRNAs (hsa-miR-24-3p, hsa-miR-885-5p, hsa-miR-101-3p, hsa-miR-34a-5p and hsa-miR-22-5p) were significantly correlated to any of its predicted miRNA targets on protein expression level (Table 1). Some genes, such as 'FYN', occurs more than once and the reason for this is that different antibodies have been used in reverse-phase protein arrays (RPPA) assay [24], and thus there will be multiple correlations for some miRNA-target pairs.

\section{MiRNA-mRNA-protein integration}

Sixteen miRNA-target gene pairs were significantly correlated at both mRNA and protein expression levels
(Table 2). In 12 out of 16 correlations, the Pearson's correlation coefficient had similar direction on mRNA and protein levels. For correlation between hsa-miR-24-3p - CDK1, the correlation is positive on mRNA expression level $(\mathrm{PCC}=0.35)$ and negative on protein expression level $(\mathrm{PCC}=-0.36)$. The opposite is observed for the correlated pairs hsa-miR-885-5p - PRKAA1, hsa-miR24-3p - KDR and hsa-miR-22-5p - PIK3CA.

\section{Functional enrichment analysis}

Predicted miRNA targets that have been filtered out as more reliable due to correlation with corresponding miRNAs were evaluated further by performing functional enrichment analysis. The most commonly occurring top GO term for all miRNA targets combined was binding (GO: 0005488) or protein binding (GO:0005515) for molecular function (Table 3), and for biological process, no specific GO term was overrepresented among the 15 miRNAs studied (Table 4). For cellular compartment (Table 5), 6 miRNAs had a top GO term connected to intracellular parts (GO:0005622 and GO:0044424). Two miRNAs (hsamiR-34a-5p and hsa-miR-885-5p) associated to pancreasrelated GO terms. Hsa-miR-34a-5p was associated to GO: 0031018; endocrine pancreas development and hsa-miR885-5p to GO:0003309; type B pancreatic cell differentiation. The miRNAs that did not have any enriched targets for GO terms or KEGG pathways were excluded from Tables $3,4,5$ and 6.

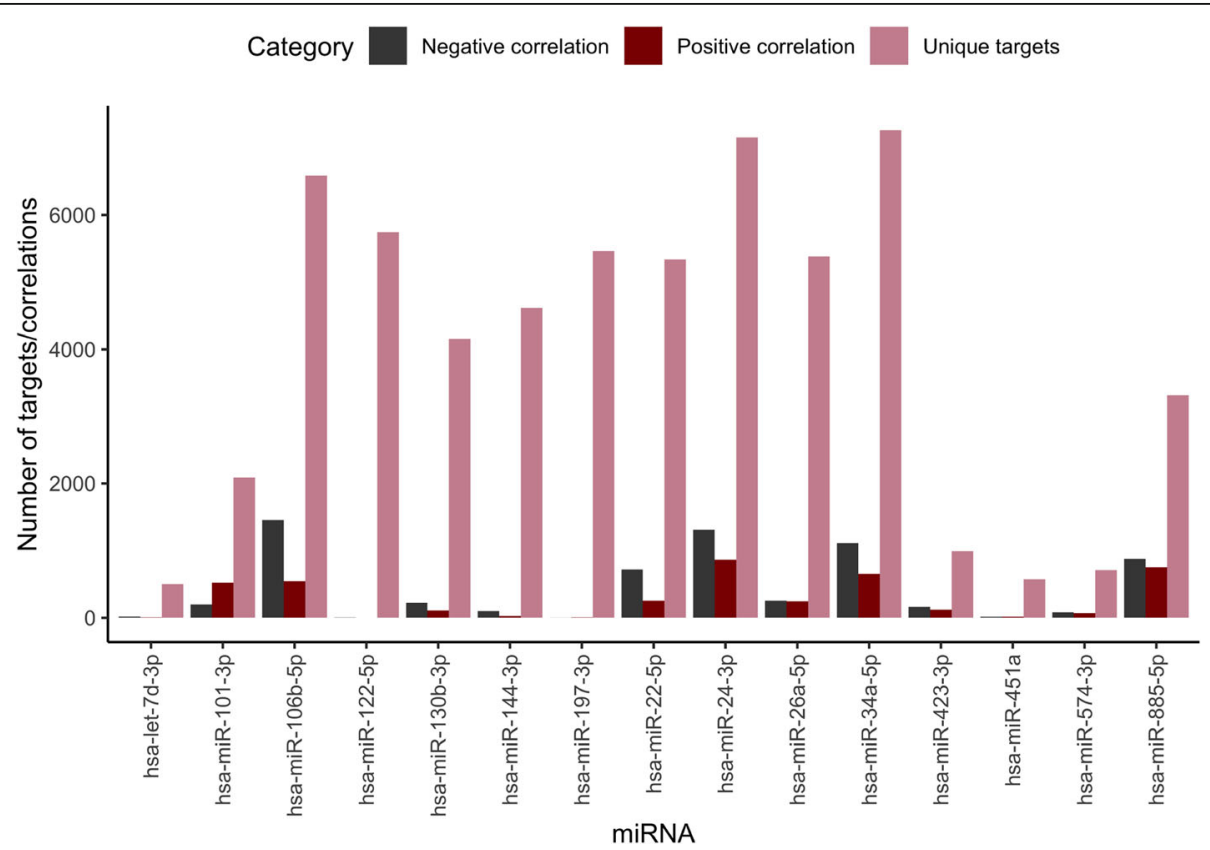

Fig. 4 Number of predicted miRNA targets, positively correlated and negatively correlated miRNA targets on mRNA level (adjusted p-value < 0.05). The $x$ axis shows each miRNA and the $y$ axis shows number of genes (predicted miRNA targets or number of correlated genes). 'Unique targets' indicate the number of miRNA targets from the union of all three miRNA target prediction databases 
Table 1 Significant correlations between miRNA and its target gene on protein level

\begin{tabular}{|c|c|c|c|}
\hline miRNA & Protein & PCC & Adjusted $P$-value \\
\hline hsa-miR-101-3p & SRSF1 & -0.32 & 0.023 \\
\hline hsa-miR-101-3p & FYN & 0.31 & 0.023 \\
\hline hsa-miR-101-3p & FYN & 0.28 & 0.048 \\
\hline hsa-miR-101-3p & PDCD4 & 0.31 & 0.023 \\
\hline hsa-miR-101-3p & MTOR & 0.33 & 0.023 \\
\hline hsa-miR-885-5p & EGFR & -0.29 & 0.035 \\
\hline hsa-miR-885-5p & RAD51 & -0.32 & 0.015 \\
\hline hsa-miR-885-5p & CDKN1B & 0.34 & 0.015 \\
\hline hsa-miR-885-5p & $\mathrm{MSH} 2$ & 0.33 & 0.015 \\
\hline hsa-miR-885-5p & GSK3B & 0.38 & 0.007 \\
\hline hsa-miR-885-5p & PRKAA1 & 0.33 & 0.015 \\
\hline hsa-miR-34a-5p & NDRG1 & -0.33 & 0.035 \\
\hline hsa-miR-34a-5p & EIF4EBP1 & -0.32 & 0.037 \\
\hline hsa-miR-34a-5p & EIF4EBP1 & -0.35 & 0.026 \\
\hline hsa-miR-34a-5p & AKT1S1 & -0.36 & 0.026 \\
\hline hsa-miR-24-3p & ASNS & -0.31 & 0.021 \\
\hline hsa-miR-24-3p & JAK2 & -0.34 & 0.014 \\
\hline hsa-miR-24-3p & ATM & -0.30 & 0.024 \\
\hline hsa-miR-24-3p & CDK1 & -0.36 & 0.014 \\
\hline hsa-miR-24-3p & YBX1 & -0.31 & 0.021 \\
\hline hsa-miR-24-3p & EIF4EBP1 & -0.31 & 0.021 \\
\hline hsa-miR-24-3p & FOXO3 & -0.28 & 0.042 \\
\hline hsa-miR-24-3p & RPS6 & -0.32 & 0.021 \\
\hline hsa-miR-24-3p & INPP4B & 0.35 & 0.014 \\
\hline hsa-miR-24-3p & $\mathrm{KDR}$ & 0.32 & 0.021 \\
\hline hsa-miR-24-3p & CCNB1 & 0.35 & 0.014 \\
\hline hsa-miR-24-3p & EGFR & 0.34 & 0.014 \\
\hline hsa-miR-24-3p & IRS1 & 0.28 & 0.042 \\
\hline hsa-miR-24-3p & ITGA2 & 0.41 & 0.004 \\
\hline hsa-miR-24-3p & CASP3 & 0.31 & 0.021 \\
\hline hsa-miR-24-3p & YWHAZ & 0.31 & 0.021 \\
\hline hsa-miR-22-5p & FYN & -0.28 & 0.049 \\
\hline hsa-miR-22-5p & SRC & -0.28 & 0.049 \\
\hline hsa-miR-22-5p & YBX1 & -0.36 & 0.007 \\
\hline hsa-miR-22-5p & YES1 & -0.28 & 0.049 \\
\hline hsa-miR-22-5p & LYN & -0.28 & 0.049 \\
\hline hsa-miR-22-5p & PTPN11 & -0.36 & 0.007 \\
\hline hsa-miR-22-5p & MYH9 & 0.28 & 0.049 \\
\hline hsa-miR-22-5p & PEA15 & 0.37 & 0.007 \\
\hline hsa-miR-22-5p & CASP3 & 0.37 & 0.007 \\
\hline hsa-miR-22-5p & PIK3CA & 0.29 & 0.049 \\
\hline hsa-miR-122-5p & MAPK14 & -0.33 & 0.042 \\
\hline hsa-miR-122-5p & EIF4EBP1 & 0.52 & 0.000004 \\
\hline
\end{tabular}

PCC Pearson's correlation coefficient
Table 2 Significant correlations on mRNA and protein expression levels

\begin{tabular}{llll}
\hline miRNA & Gene & PCC (mRNA level) & PCC (protein level) \\
\hline hsa-miR-101-3p & FYN & 0.33 & 0.31 \\
hsa-miR-101-3p & FYN & 0.33 & 0.28 \\
hsa-miR-101-3p & PDCD4 & 0.40 & 0.31 \\
hsa-miR-885-5p & CDKN1B & 0.25 & 0.34 \\
hsa-miR-885-5p & EGFR & -0.46 & -0.29 \\
hsa-miR-885-5p & PRKAA1 & -0.28 & 0.33 \\
hsa-miR-885-5p & RAD51 & -0.47 & -0.32 \\
hsa-miR-24-3p & CCNB1 & 0.33 & 0.35 \\
hsa-miR-24-3p & CDK1 & 0.35 & -0.36 \\
hsa-miR-24-3p & INPP4B & 0.37 & 0.35 \\
hsa-miR-24-3p & ITGA2 & 0.34 & 0.41 \\
hsa-miR-24-3p & KDR & -0.24 & 0.32 \\
hsa-miR-24-3p & RPS6 & -0.25 & -0.32 \\
hsa-miR-24-3p & YWHAZ & 0.51 & 0.31 \\
hsa-miR-22-5p & PIK3CA & -0.20 & 0.29 \\
hsa-miR-22-5p & PTPN11 & -0.21 & -0.36 \\
\hline
\end{tabular}

PCC Pearson's correlation coefficient

The top KEGG pathway varied among the miRNAs but the Rap1 signaling pathway (path:hsa04015) was the only one that occurred more than once (Table 6). No GO term or KEGG pathway enrichment was found for correlated miRNA targets of hsa-let-7d-3p, hsa-miR122-5p, hsa-miR-197-3p or hsa-miR-451a.

\section{Overlap of miRNAs}

Initially, we were interested to see if there are any shared targets between our panel of 15 differentially expressed miRNAs. No overlap of predicted miRNA targets was detected for all 15 miRNAs combined. However, by studying the established list of their enriched KEGG pathways, we could determine four miRNAs (hsa-miR22-5p, hsa-miR-24-3p, hsa-miR-106b-5p and hsa-miR885-5p) associated to hsa0512 'Pancreatic cancer' (see Additional files 1, 2, 3 and 4). Based on this finding, miRNA target genes shared between these four miRNAs were studied further. Sixteen overlapping significantly correlated miRNA target genes were identified (Table 7). Nuclear factor I B (NFIB) shows similar correlation coefficients between these four miRNAs.

\section{Survival analysis}

Due to many identified correlations observed between the miRNAs and their target genes suggesting a regulatory role in pancreatic cancer, we further studied the fifteen miRNAs as prognostic biomarkers by Kaplan-Meier survival analysis. The median was used as cut-off and hsamiR-885-5p was found to be significantly correlated to 
Table 3 Top significant molecular function GO term for each miRNA. 'Count' represents number of miRNA targets enriched

\begin{tabular}{llll}
\hline miRNA & GO term & Count & $P$-value \\
\hline hsa-let-7d-3p & GO:0140110 transcription regulator activity & 8 & 0.004 \\
hsa-miR-101-3p & GO:0005488 binding & 622 & $<0.001$ \\
hsa-miR-106b-5p & GO:0005488 binding & 1633 & 0.001 \\
hsa-miR-130b-3p & GO:0005024 transforming growth factor beta-activated receptor activity & 5 & $<.001$ \\
hsa-miR-144-3p & GO:0005515 protein binding & 91 & 0.001 \\
hsa-miR-197-3p & GO:1901363 heterocyclic compound binding & 5 & 0.011 \\
hsa-miR-22-5p & GO:0005515 protein binding & 695 & $<.001$ \\
hsa-miR-24-3p & GO:0005515 protein binding & 1447 & $<.001$ \\
hsa-miR-26a-5p & GO:0005488 binding & 423 \\
hsa-miR-34a-5p & GO:0005488 binding & 1442 \\
hsa-miR-423-3p & GO:0044212 transcription regulatory region DNA binding & 31 & $<.001$ \\
hsa-miR-451a & GO:0005515 protein binding & 23 & 0.001 \\
hsa-miR-574-3p & GO:0003674 molecular_function & 147 & 0.001 \\
hsa-miR-885-5p & GO:0005488 binding & 1335 & 0.011 \\
\hline
\end{tabular}

NA not applicable

survival (Fig. 5, nominal $p$-value $=0.032$ ). However, after adjusting for multiple hypothesis testing, none of the 15 miRNAs analyzed was significant for overall survival in the TCGA-PAAD dataset (Additional file 6: Figure S2).

\section{Network analysis of hsa-miR-885-5p targets}

The correlated miRNA target genes can be used for other downstream analyses, one example used here is network analyses. For this, we used hsa-miR-885-5p as an example and analyzed negatively and positively correlated targets separately. Hub genes were extracted (Fig. 6), where the top 10 connected proteins are shown together with the rank of each hub gene. ClueGO and CluePedia were used to visualize the interplay between significant KEGG pathways and to see which genes connect the pathways (Fig. 7). Negatively and positively correlated gene targets were handled separately. To narrow down the number of targets analyzed, a correlation coefficient cut-off of 0.4 (positive correlations) or -0.4 (negative correlations) was used. Consequently, only target genes correlating on mRNA expression levels were included in these analyses as the targets correlated on protein expression levels were below this cutoff. Three genes are shared between many pathways in the negatively correlated network (Fig. 7a); EGFR (9 pathways), CTNNB1 (10 pathways) and NRAS (9 pathways).

\section{Comparison to other tools}

MiRFA has the strength of combining miRNA target prediction and correlation analyses (positive and negative

Table 4 Top significant biological process GO term for each miRNA. 'Count' represents number of miRNA targets enriched

\begin{tabular}{|c|c|c|c|}
\hline miRNA & GO term & Count & $P$-value \\
\hline hsa-let-7d-3p & GO:0051962 positive regulation of nervous system development & 5 & 0.001 \\
\hline hsa-miR-101-3p & GO:0019219 regulation of nucleobase-containing compound metabolic process & 249 & $<0.001$ \\
\hline hsa-miR-106b-5p & GO:0007399 nervous system development & 356 & $<0.001$ \\
\hline hsa-miR-130b-3p & GO:0046834 lipid phosphorylation & 11 & $<0.001$ \\
\hline hsa-miR-144-3p & GO:0072659 protein localization to plasma membrane & 7 & 0.001 \\
\hline hsa-miR-22-5p & GO:0051641 cellular localization & 253 & $<0.001$ \\
\hline hsa-miR-24-3p & GO:0023051 regulation of signaling & 516 & $<0.001$ \\
\hline hsa-miR-26a-5p & G0:0002009 morphogenesis of an epithelium & 28 & $<0.001$ \\
\hline hsa-miR-34a-5p & GO:0050794 regulation of cellular process & 1084 & $<0.001$ \\
\hline hsa-miR-423-3p & GO:0065009 regulation of molecular function & 77 & $<0.001$ \\
\hline hsa-miR-451a & GO:0000165 MAPK cascade & 8 & $<0.001$ \\
\hline hsa-miR-574-3p & GO:0071495 cellular response to endogenous stimulus & 22 & 0.001 \\
\hline hsa-miR-885-5p & GO:0031323 regulation of cellular metabolic process & 603 & $<0.001$ \\
\hline
\end{tabular}


Table 5 Top significant cellular component GO term for each miRNA. 'Count' represents number of miRNA targets enriched

\begin{tabular}{llll}
\hline miRNA & GO term & Count & $P$-value \\
\hline hsa-let-7d-3p & GO:0044459 plasma membrane part & 8 & 0.026 \\
hsa-miR-101-3p & GO:0005654 nucleoplasm & $224<0.001$ \\
hsa-miR-106b-5p & GO:0005622 intracellular & $1630<0.001$ \\
hsa-miR-130b-3p & GO:0044444 cytoplasmic part & $196<0.001$ \\
hsa-miR-144-3p & GO:0070161 anchoring junction & 14 & $<0.001$ \\
hsa-miR-22-5p & GO:0044424 intracellular part & $828<0.001$ \\
hsa-miR-24-3p & GO:0005737 cytoplasm & $1388<0.001$ \\
hsa-miR-26a-5p & GO:0044424 intracellular part & $427<0.001$ \\
hsa-miR-34a-5p & GO:0005622 intracellular & $1431<0.001$ \\
hsa-miR-423-3p & GO:0005737 cytoplasm & $191<0.001$ \\
hsa-miR-451a & GO:0031252 cell leading edge & 6 & $<0.001$ \\
hsa-miR-574-3p & GO:0044424 intracellular part & $132<0.001$ \\
hsa-miR-885-5p & GO:0005622 intracellular & $1322<0.001$ \\
\hline NA not applicable & & &
\end{tabular}

correlations) on both mRNA and protein expression levels. Furthermore, miRFA includes mature miRNA expression in the correlation analyses and performs functional enrichment of the correlated targets. Another strength of our tool is that it is also web-based. We compared our tools to others that perform miRNA functional analysis or functional annotations (Table 8). MiRFA and miRCancerdb [16] are both available as $\mathrm{R}$ packages and web-based tools. MultiMiR [12], RBiomirGS [13], MiRComb [14] and miRLab [15] are only available as $\mathrm{R}$ packages, whereas MiRpath [18], miEAA [28], TAM [29] and GeneTrail2 [30] are webbased resources. Four tools (miRFA, miRCancerdb, miRComb, miRLab) take into account correlation analysis in combination with miRNA target prediction. Our tool does not provide information on miRNA annotation such as miRNA clusters or families that can be obtained using miEAA or TAM tools. Furthermore, our tool does not offer a functional analysis of precursor hairpin miRNAs and is restricted to pancreatic cancer in its current form.

In addition to the feature comparison between tools, shown in Table 8, we analyzed our list of 15 miRNAs (Tables 9 and 10) in miRCancerdb. Since MiRCancerdb does not provide the option to analyze functional enrichment, this feature was not considered for a comparison. In order to obtain all correlated targets in miRCancerdb, we set a threshold to 10,000 correlations, and select parameters 'PAAD' for TCGA study code, 'Targets only' for feature type and both direction of correlation with an absolute minimum of 0 for correlation. MiRCancerdb has filtered out correlations less than 0.1 so these correlations were not included in our comparison since we used the web-based tool [16]. Since miRCancerdb is built with precursor miRNAs, we used the precursor names of our 15 miRNAs. To benchmark miRCancerdb with our tool, we used the genes list from KEGG pathway hsa05212 pancreatic cancer (75 genes) and counted how many pancreatic cancer-related genes were obtained in the two tools (Tables 9 and 10). MiRNAs with 0 correlated targets in both tools were excluded from the tables. MiRCancerdb generates some overlap of correlated targets between has-mir-144 (miRCancerdb) and hsa-miR-144-3p (miRFA), but we can also find overlap of correlated targets between mir-144 (miRCancerdb) and the other mature miRNA; hsa-miR-144-5p (miRFA).

\section{Discussion}

The aim of this study was to build a bioinformatics pipeline for miRNA functional analysis and correlation analyses for in silico evaluation (Fig. 1). Expression data of mature miRNA isoforms was included in correlation analyses since the differentially expressed mature miRNAs were used as input miRNAs in the pipeline (Fig. 2). Many of the TCGA samples showed expression in hsamiR-144-3p and not in hsa-miR-144-5p. Relying on the

Table 6 Top significant KEGG pathway for each miRNA. 'Count' represents number of miRNA targets enriched

\begin{tabular}{|c|c|c|c|}
\hline miRNA & Pathway & Count & $P$-value \\
\hline hsa-miR-101-3p & path:hsa04070 Phosphatidylinositol signaling system & 13 & $<0.001$ \\
\hline hsa-miR-106b-5p & path:hsa04015 Rap1 signaling pathway & 46 & $<0.001$ \\
\hline hsa-miR-130b-3p & path:hsa04350 TGF-beta signaling pathway & 8 & $<0.001$ \\
\hline hsa-miR-144-3p & path:hsa05200 Pathways in cancer & 7 & 0.041 \\
\hline hsa-miR-22-5p & path:hsa04360 Axon guidance & 23 & $<0.001$ \\
\hline hsa-miR-24-3p & path:hsa05100 Bacterial invasion of epithelial cells & 24 & $<0.001$ \\
\hline hsa-miR-26a-5p & path:hsa04510 Focal adhesion & 15 & $<0.001$ \\
\hline hsa-miR-34a-5p & path:hsa04810 Regulation of actin cytoskeleton & 38 & $<0.001$ \\
\hline hsa-miR-423-3p & path:hsa04015 Rap1 signaling pathway & 13 & $<0.001$ \\
\hline hsa-miR-574-3p & path:hsa01522 Endocrine resistance & 6 & $<0.001$ \\
\hline hsa-miR-885-5p & path:hsa05160 Hepatitis C & 28 & $<0.001$ \\
\hline
\end{tabular}


Table 7 Pearson's correlation coefficient shown for overlapping predicted miRNA target genes of four miRNAs

\begin{tabular}{|c|c|c|c|c|}
\hline Gene & hsa-miR-22-5p & hsa-miR-24-3p & hsa-miR-106b-5p & hsa-miR-885-5p \\
\hline MAP 1B & -0.24 & -0.20 & -0.40 & 0.33 \\
\hline NFIB & -0.19 & -0.33 & -0.35 & -0.29 \\
\hline REV3L & -0.22 & -0.28 & -0.30 & 0.18 \\
\hline LONRF2 & -0.24 & -0.26 & -0.34 & 0.34 \\
\hline TMTC1 & -0.24 & -0.22 & -0.21 & 0.21 \\
\hline MSANTD4 & -0.30 & -0.18 & -0.29 & 0.22 \\
\hline HCN1 & -0.23 & -0.24 & -0.33 & 0.17 \\
\hline SIK2 & -0.23 & -0.21 & -0.22 & 0.38 \\
\hline GABRG2 & -0.22 & -0.19 & -0.24 & 0.33 \\
\hline DCX & -0.20 & -0.21 & -0.22 & 0.25 \\
\hline KCND3 & -0.21 & -0.28 & -0.35 & 0.28 \\
\hline NTRK2 & -0.19 & -0.28 & -0.23 & 0.19 \\
\hline CNTNAP5 & -0.21 & -0.24 & -0.28 & 0.21 \\
\hline FRRS1L & -0.32 & -0.22 & -0.36 & 0.37 \\
\hline MGAT4C & -0.21 & -0.31 & -0.28 & 0.44 \\
\hline TMEM134 & 0.32 & 0.18 & 0.27 & -0.19 \\
\hline
\end{tabular}

precursor hsa-mir-144 expression would have caused false-positive expression values as the precursor hsamir-144 expression pattern is more similar to the expression of the $-5 p$ mature miRNA in this case. The pipeline generated miRNA targets, correlated targets, enriched GO terms and KEGG pathways for 15 miRNAs. This study utilized input miRNAs detected in plasma samples of PDAC patients [7], whereas the expression data used for correlation analyses originated from tumor tissue. The circulating miRNAs could be a leakage from the tumor or a systemic response to the cancer state.

MiRNA target prediction tends to generate a lot of false-positives [19], which is why correlation analyses

\section{hsa-miR-885-5p}

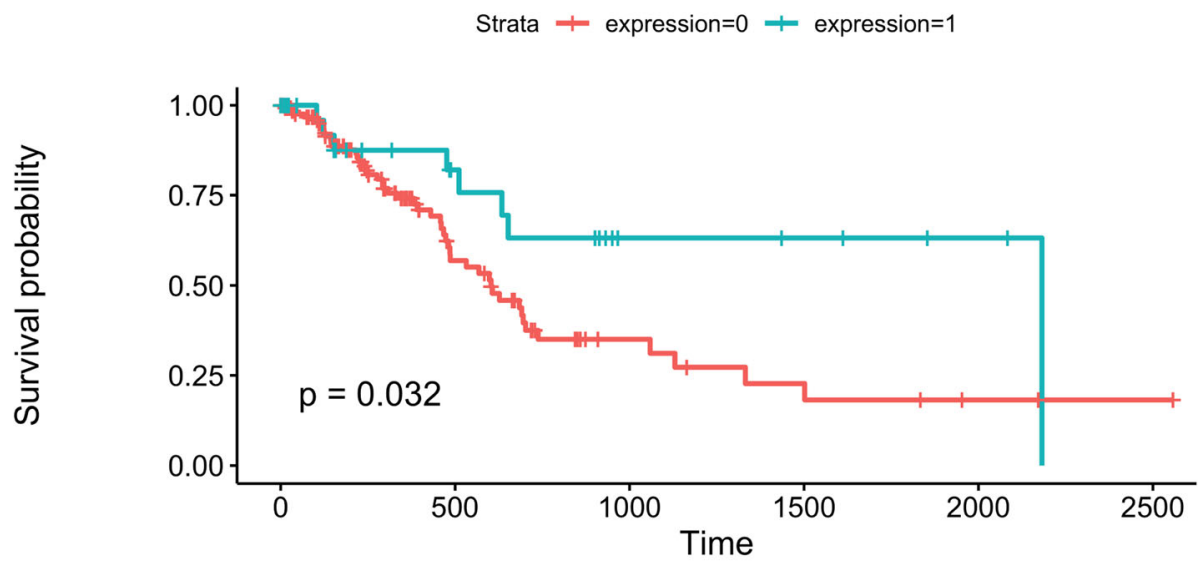

Number at risk

\begin{tabular}{|c|c|c|c|c|c|c|}
\hline \multirow{2}{*}{ 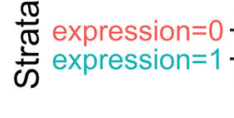 } & $\begin{array}{c}146 \\
31 \\
\end{array}$ & $\begin{array}{l}32 \\
13 \\
\end{array}$ & $\begin{array}{l}9 \\
5\end{array}$ & $\begin{array}{l}5 \\
4 \\
\end{array}$ & $\begin{array}{l}2 \\
2\end{array}$ & $\begin{array}{l}1 \\
0\end{array}$ \\
\hline & 0 & 500 & 1000 & 1500 & 2000 & 2500 \\
\hline
\end{tabular}

Fig. 5 Overall survival for hsa-miR-885-5p using median $\log 2(r p m+1)$ expression as cut-off. Expression $=0$ is the group that has a value below median and expression $=1$ is the group that has a value above median. The nominal $p$-value is displayed $(p=0.032)$, but was not significant after multiple hypothesis correction using Benjamini-Hochberg 

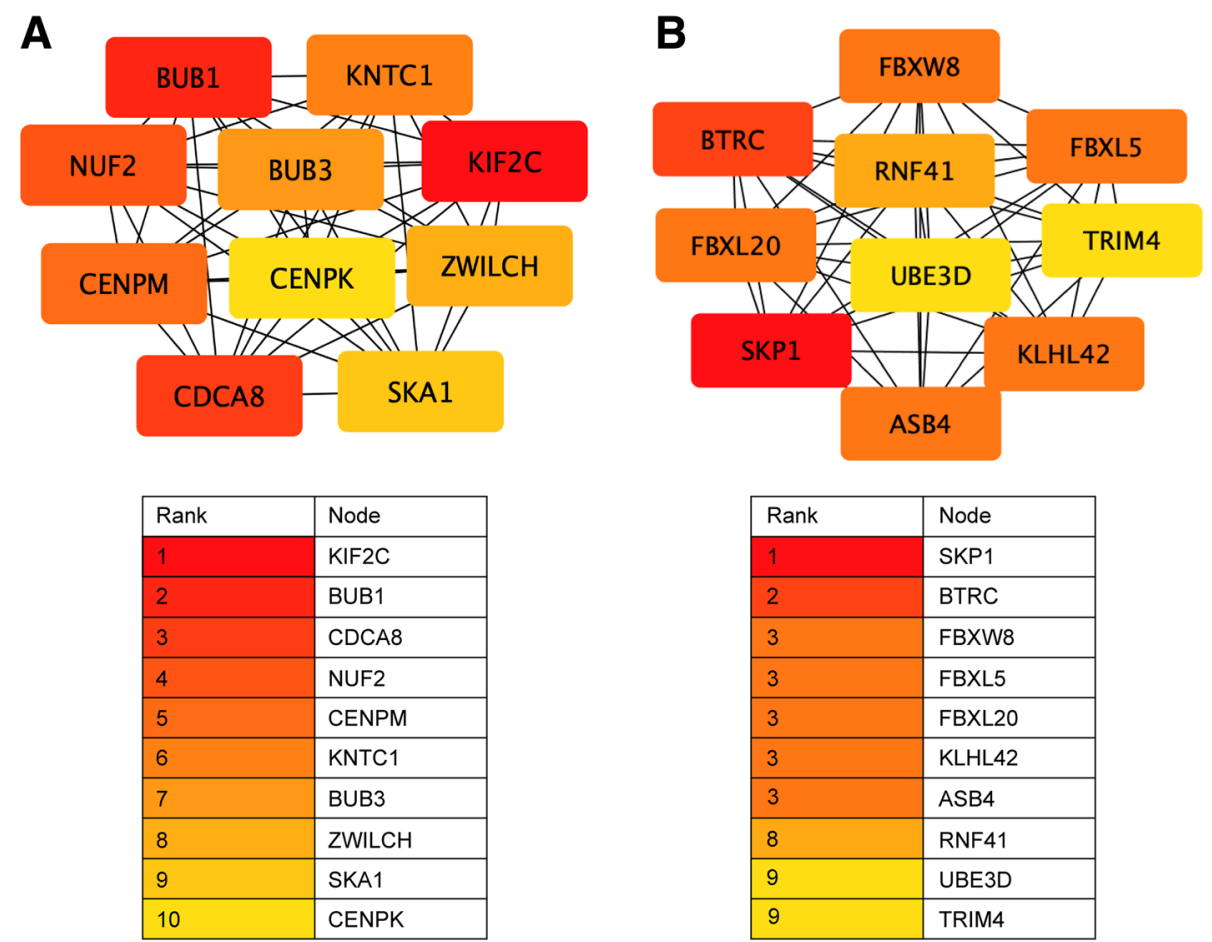

Fig. 6 Hub genes for hsa-miR-885-5p. Top 10 hub genes and their ranks are shown for negatively correlated (a) and positively correlated (b) targets

between each miRNA and its predicted targets were performed as an in silico evaluation. Correlation analysis is one way of determining the dependency between two variables [31] and was applied on expression levels of miRNA and its target genes on both mRNA and protein levels in this study. Correlation analyses do not automatically indicate that the dependency is direct, however, since the miRNA-gene pairs were predicted to interact, it gives a stronger support for a miRNA-mediated regulation effect. Including the correlation analyses saves time in post-processing steps of extracting interesting miRNA target candidates since the output list of interesting candidates becomes shorter after in silico evaluation.

The number of correlated miRNA-target pairs (on mRNA expression level) were not associated to the number of targets predicted by the databases (Fig. 4), i.e. that a higher number of predicted miRNA targets would automatically generate a higher number of significant correlations. In the study by Seo et al. [21], protein expression data was included in the correlations as miRNA-mediated regulation acts post-transcriptionally and thus mainly affects the protein expression levels. MiRNAs regulate their targets by degradation or repression and an effect on the protein level might not always be visible on mRNA level [4]. Hence, when possible, the protein expression levels are useful in correlation-based in silico evaluation. One limitation for using correlation analyses based on mRNA and protein expression data is the risk for false negatives, due to missing expression data for some predicted targets, especially for the protein expression data in this case. TCPA provide expression data for around 200 proteins and resulted in only 43 significant correlations (Table 1) as compared to a total of 10,754 correlated miRNA-target pairs on mRNA expression level (see Additional file 5) accounting for all 15 miRNAs. Hence, there is a need for more highthroughput proteomics for miRNA functional analysis purposes. No feature was included in the pipeline to show which targets were not available among mRNA or protein expression data.

A possible drawback of our pipeline is introduction of false positive correlations between miRNAs and its targets. The trade-off between specificity and sensitivity in biomarker discovery is always of great importance. Our intention with the proposed pipeline is to provide a tool that will support an early phase of exploratory research on candidate biomarkers in heterogeneous diseases. Given that premise, we suggest that the value of finding novel important biomarkers may override the concern with introducing false connections.

Kaplan-Meier survival analysis suggests that hsa-miR885-5p may act as a tumor suppressor in PDAC (Fig. 5). This is supported by previous functional studies of hsamiR-885-5p. Hsa-miR-885-5p was previously identified 


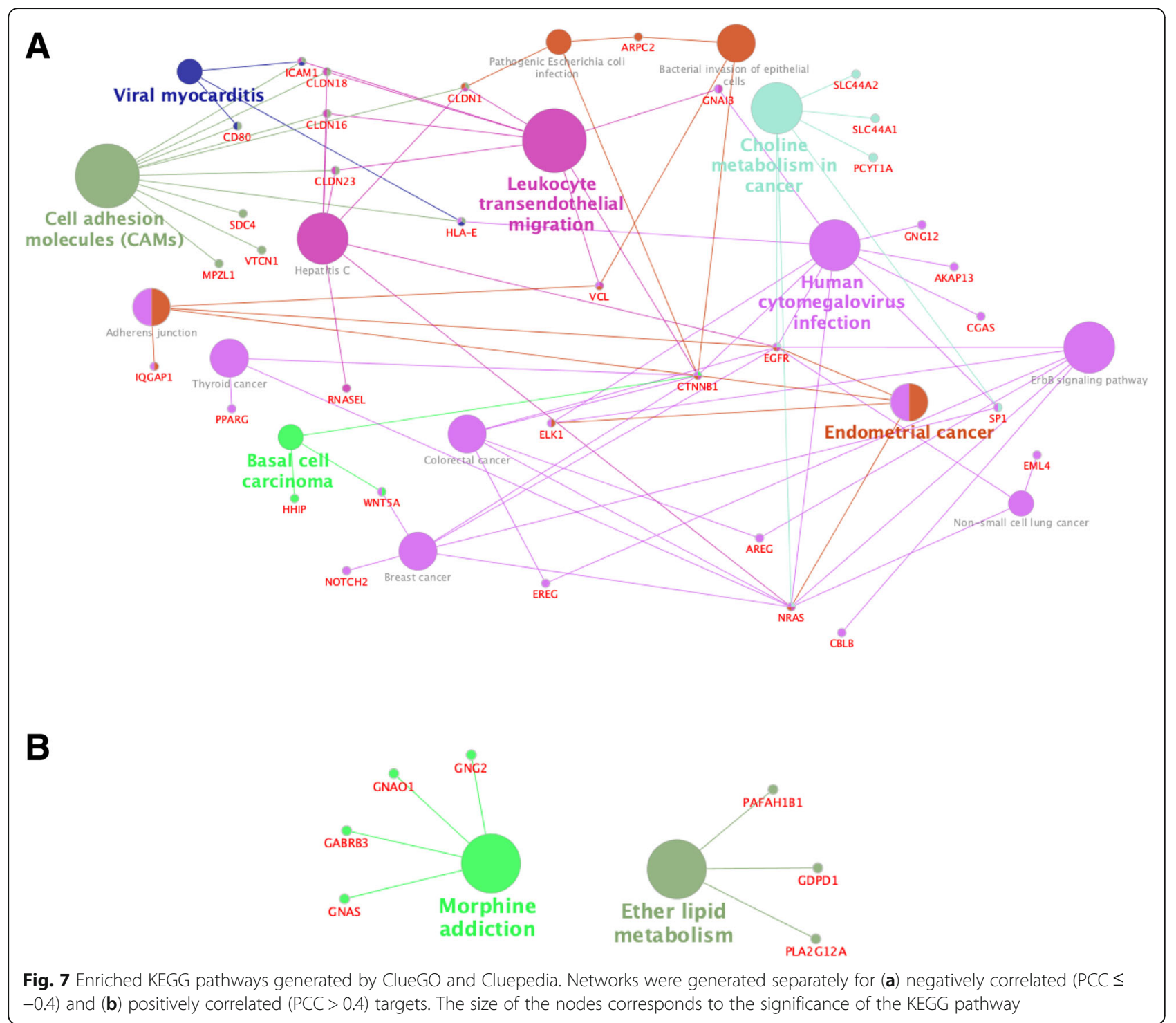

to act as a tumor suppressor in hepatocellular carcinoma [32] and hepatoblastoma [33] by targeting $\beta$-catenin. Furthermore, a decay in expression correlated to a more progressed hepatocellular carcinoma by correlation to tumor-node-metastasis (TNM) stages [32]. In this study, $\beta$-catenin (CTNNB1) was predicted as a target for hsamiR-885-5p by TargetScan v7.1 and DIANA-microTCDS, and a significant negative correlation on mRNA level $(P C C=-0.46)$ was identified. In addition, hsa-miR$885-5 p$ has previously been found up-regulated in liver metastases compared to the primary tumor in colorectal cancer [34], and a regulation involving its predicted target CPEB2 has been suggested [24]. CPEB2 was identified as a target for hsa-miR-885-5p by TargetScan and DIANAmicroT-CDS in this study but was not significantly correlated. In addition, several studies support hsa-miR-885-5p as a circulating biomarker in PDAC $[7,9,35,36]$.
MiRNA target prediction lacks information about other factors that could affect the extent of miRNAmediated regulation. Hence, further validation is needed to increase the reliability of the identified targets and extract genes of interest in a disease-specific context, in this case pancreatic cancer. Experimental validation and additional bioinformatics analyses can be applied to correlated miRNA targets, such as functional enrichment (Tables 3, 4, 5 and 6) and network analyses (Figs. 6 and 7). Our network analysis resulted in the top ranked hub genes KIF2C (kinesin family member $2 \mathrm{C}$, also known as MCAK) for negatively correlated targets (Fig. 6a) and SKP1 (S-phase kinase associated protein 1) for positively correlated targets (Fig. 6b). KIF2C is involved in mitosis by associating to the centromere [37]. BUB1 (BUB1 mitotic checkpoint serine/threonine kinase) was found as top 2 for the negatively correlated targets and is active in a complex 
Table 8 Comparison of miRFA to other available tools for functional analysis of miRNAs

\begin{tabular}{|c|c|c|c|c|c|c|c|c|c|c|c|}
\hline \multirow[t]{2}{*}{ Feature } & & \multicolumn{10}{|l|}{ Tool } \\
\hline & & miRFA & miRCancerdb & multiMiR & RBiomirGS & MiRComb & miRLab & miRpath & miEAA & TAM & GeneTrail2 \\
\hline Platform & & $\begin{array}{l}\text { R/Web- } \\
\text { based }\end{array}$ & R/Web-based & $R$ & $R$ & $\mathrm{R}$ & $\mathrm{R}$ & $\begin{array}{l}\text { Web- } \\
\text { based }\end{array}$ & $\begin{array}{l}\text { Web- } \\
\text { based }\end{array}$ & $\begin{array}{l}\text { Web- } \\
\text { based }\end{array}$ & $\begin{array}{l}\text { Web- } \\
\text { based }\end{array}$ \\
\hline \multirow{2}{*}{$\begin{array}{l}\text { MiRNA input/ } \\
\text { expression }\end{array}$} & Mature miRNA & $\checkmark$ & & $\checkmark$ & $\checkmark$ & $\checkmark$ & a & $\checkmark$ & $\checkmark$ & & $\checkmark$ \\
\hline & Precursor miRNA & & $\checkmark$ & & & $\checkmark$ & $\checkmark$ & & $\checkmark$ & $\checkmark$ & \\
\hline \multirow[t]{2}{*}{$\begin{array}{l}\text { MiRNA target } \\
\text { prediction database }\end{array}$} & $\begin{array}{l}\text { Experimentally } \\
\text { validated } \\
\text { database(s) }\end{array}$ & $\checkmark$ & & $\checkmark$ & $\checkmark$ & & $\checkmark$ & $\checkmark$ & $\checkmark$ & & $\checkmark$ \\
\hline & $\begin{array}{l}\text { Target prediction } \\
\text { database(s) }\end{array}$ & $\checkmark$ & $\checkmark$ & $\checkmark$ & $\checkmark$ & $\checkmark$ & $\checkmark$ & $\checkmark$ & & & \\
\hline \multirow{4}{*}{$\begin{array}{l}\text { Correlation analysis } \\
\text { expression levels }\end{array}$} & mRNA expression & $\checkmark$ & $\checkmark$ & & & $\checkmark$ & $\checkmark$ & & & & \\
\hline & Protein expression & $\checkmark$ & $\checkmark$ & & & & & & & & \\
\hline & Negative correlation & $\checkmark$ & $\checkmark$ & & & $\checkmark$ & $\checkmark^{b}$ & & & & \\
\hline & Positive correlation & $\checkmark$ & $\checkmark$ & & & & & & & & \\
\hline $\begin{array}{l}\text { Functional } \\
\text { enrichment }\end{array}$ & & $\checkmark$ & & & $\checkmark$ & $\checkmark$ & $\checkmark$ & $\checkmark$ & $\checkmark$ & $\checkmark$ & $\checkmark$ \\
\hline Disease-specific & & $\checkmark$ & $\checkmark$ & $\checkmark$ & & & $\checkmark$ & & & & \\
\hline miRNA annotation ${ }^{c}$ & & & & & & & & & $\checkmark$ & $\checkmark$ & $\checkmark$ \\
\hline
\end{tabular}

${ }^{a}$ Based on if the user implements built in TCGA retriever

${ }^{b}$ Negative correlations are ranked at the top

' Includes cluster, family, functions, diseases, tissue specificity

together with BUB3 [38], which is also among the hub genes for negatively correlated targets (Fig. 6a). This complex (BUB1/BUBR1/BUB3) is necessary for correct balance of kinase-phosphatase balance during mitosis and has been proven to have a role in chromosome instability and tumor progression as well [38]. Skp1 has been suggested to have a role in various cancer forms by contributing to active oncogenic (Skp1)-Cullin1-F-box protein (SCF) complexes [39]. SCF complexes is the best characterized E3 ligases and are involved in protein degradation.

NRAS, EGFR and CTNNB1 ( $\beta$-catenin) were found to overlap between many enriched KEGG pathways for

Table 9 Comparison of correlated targets between miRCancerdb and miRFA on mRNA level. KEGG genes refer to genes included in KEGG pathway pancreatic cancer

\begin{tabular}{|c|c|c|c|c|c|c|c|}
\hline \multicolumn{4}{|l|}{ miRCancerdb } & \multicolumn{4}{|l|}{ miRFA } \\
\hline Input & Cor mRNA & KEGG genes & Percentage (\%) & Input & Cor mRNA & KEGG genes & Percentage (\%) \\
\hline hsa-mir-144 & 284 & 1 & 0.4 & hsa-miR-144-3p & 127 & 19 & 15.0 \\
\hline hsa-mir-106b & 458 & 6 & 1.3 & hsa-miR-106b-5p & 2005 & 14 & 0.7 \\
\hline hsa-mir-451a & 0 & NA & NA & hsa-miR-451a & 29 & 0 & 0 \\
\hline hsa-mir-101 & 0 & NA & NA & hsa-miR-101-3p & 713 & 5 & 0.7 \\
\hline hsa-mir-26a & 0 & NA & NA & hsa-miR-26a-5p & 508 & 0 & 0 \\
\hline hsa-mir-574 & 0 & NA & NA & hsa-miR-574-3p & 156 & 2 & 1.3 \\
\hline hsa-mir-885 & 0 & NA & NA & hsa-miR-885-5p & 1642 & 12 & 0.7 \\
\hline hsa-mir-130b & 277 & 3 & 1.1 & hsa-miR-130b-3p & 333 & 3 & 0.9 \\
\hline hsa-mir-34a & 176 & 2 & 1.1 & hsa-miR-34a-5p & 1774 & 10 & 0.6 \\
\hline hsa-mir-24 & 0 & NA & NA & hsa-miR-24-3p & 2179 & 15 & 0.7 \\
\hline hsa-mir-22 & 261 & 4 & 1.5 & hsa-miR-22-5p & 977 & 8 & 0.8 \\
\hline hsa-let-7d & 443 & 5 & 1.1 & hsa-let-7d-3p & 28 & 0 & 0 \\
\hline hsa-mir-197 & 57 & 0 & 0 & hsa-miR-197-3p & 7 & 0 & 0 \\
\hline hsa-mir-423 & 0 & NA & NA & hsa-miR-423-3p & 288 & 2 & 0.7 \\
\hline hsa-mir-122 & 50 & 0 & 0 & hsa-miR-122-5p & 3 & 0 & 0 \\
\hline
\end{tabular}

Cor mRNA = number of correlated miRNA - targets on mRNA level, KEGG genes = number of genes associated to KEGG pathway hsa05212 pancreatic cancer, percentage $=$ percentage of KEGG genes compared to total number of correlated targets, NA = not available 
Table 10 Comparison of correlated targets between miRCancerdb and miRFA on protein expression level

\begin{tabular}{|c|c|c|c|c|c|c|c|c|c|}
\hline \multicolumn{5}{|l|}{ miRCancerdb } & \multicolumn{5}{|l|}{ miRFA } \\
\hline Input & $\begin{array}{l}\text { Cor } \\
\text { protein }\end{array}$ & $\begin{array}{l}\text { KEGG } \\
\text { genes }\end{array}$ & $\begin{array}{l}\text { Percentage } \\
(\%)\end{array}$ & Gene names & Input & $\begin{array}{l}\text { Cor } \\
\text { protein }\end{array}$ & $\begin{array}{l}\text { KEGG } \\
\text { genes }\end{array}$ & $\begin{array}{l}\text { Percentage } \\
(\%)\end{array}$ & $\begin{array}{l}\text { Gene } \\
\text { names }\end{array}$ \\
\hline hsa-mir-144 & 5 & 0 & 0 & NA & hsa-miR-144-3p & 0 & NA & NA & NA \\
\hline $\begin{array}{l}\text { hsa-mir- } \\
\text { 106b }\end{array}$ & 3 & 2 & 67 & AKT1/2/3, RB1 & $\begin{array}{l}\text { hsa-miR-106b- } \\
5 p\end{array}$ & 0 & NA & NA & NA \\
\hline hsa-mir-101 & 0 & NA & NA & NA & hsa-miR-101-3p & 5 & 1 & 20 & MTOR \\
\hline hsa-mir-885 & 0 & NA & NA & NA & hsa-miR-885-5p & 6 & 2 & 33 & $\begin{array}{l}\text { EGFR, } \\
\text { RAD51 }\end{array}$ \\
\hline $\begin{array}{l}\text { hsa-mir- } \\
\text { 130b }\end{array}$ & 3 & 1 & 33 & SMAD4 & $\begin{array}{l}\text { hsa-miR-130b- } \\
3 p\end{array}$ & 0 & NA & NA & NA \\
\hline hsa-mir-34a & 2 & 1 & 50 & SMAD4 & hsa-miR-34a-5p & 4 & 0 & 0 & NA \\
\hline hsa-mir-24 & 0 & NA & NA & NA & hsa-miR-24-3p & 16 & 1 & 6 & EGFR \\
\hline hsa-mir-22 & 3 & 2 & 67 & AKT1/2/3, CDKN1A & hsa-miR-22-5p & 10 & 1 & 10 & PIK3CA \\
\hline hsa-let-7d & 4 & 3 & 75 & $\begin{array}{l}\text { AKT1/2/3, CDKN1A, } \\
\text { RB1 }\end{array}$ & hsa-let-7d-3p & 0 & NA & NA & NA \\
\hline hsa-mir-122 & 0 & NA & NA & NA & hsa-miR-122-5p & 2 & 0 & 0 & NA \\
\hline
\end{tabular}

Cor protein = number of correlated miRNA - targets on mRNA level, KEGG genes = number of genes associated to KEGG pathway hsa05212 pancreatic cancer, percentage $=$ percentage of KEGG genes compared to total number of correlated targets, NA $=$ not available

hsa-miR-885-5p negatively correlated targets (Fig. 7a). Expression of these three genes in a PDAC context has been studied previously. Overexpression of EGFR is detected in a fraction of PDAC patients with a range of $30.4-64.2 \%$ in different PDAC cohorts [40-45]. $\beta$ catenin expression in PDAC tissue was previously reported as a prognostic marker, where a high expression using immunohistochemistry staining predicted longer survival [46]. NRAS has also been suggested as a protective biomarker in PDAC as assessed by immunohistochemistry [47]. A high fraction of PDAC patients harbor KRAS mutations,

91\% in the TCGA-PAAD dataset, whereas NRAS mutation are rare [47]. KRAS was identified as a miRNA target and significantly correlated $(\mathrm{PCC}=-$ 0.19) with hsa-miR-885-5p (see Additional file 5). NRAS showed stronger correlation to hsa-miR-885-5p $(\mathrm{PCC}=-0.42)$ than KRAS.

As highlighted in the comparison between our tool and other miRNA functional analysis tools, many resources exist for this type of analysis (Table 8). Although many tools resemble each other in terms of provided features, they still have significant differences and make up a very broad toolkit to apply in miRNA functional analysis. Hence, we identified an important gap to fill by developing a tool for correlating both mRNA and protein expression levels using mature miRNA isoforms expression levels. The mechanisms behind miRNAmediated regulation are highly complex and act in a disease- or tissue-specific manner [48].

MiRFA was compared to miRCancerdb with regards to our list of 15 miRNAs (Tables 9 and 10). It is difficult to assess the performances of these tools in terms of prediction accuracy, since we do not know true miRNA targets in pancreatic cancer. Still, to be able to perform an objective comparison, we selected a benchmarking dataset consisting of 75 genes found in KEGG pathway hsa05212 pancreatic cancer. We calculated the fraction of pancreatic cancer pathway-associated genes identified by each of the tool (miRCancerdb and miRFA, Tables 9 and 10). The main difference between these tools is that miRCancerdb implements the miRNA-seq data from TCGA-PAAD while miRFA implements pre-processed mature miRNA isoform quantification expression data. The correlation dataset available in miRCancerdb is restricted to correlations above 0.1 , whereas we have applied a threshold of adjusted $p$-value $<0.05$.

Correlations were not obtained for all miRNAs in miRCancerdb. The targetscan $\mathrm{R}$ package targetscan. Hs.eg.db [49] was used to obtain targetscan targets in miRCancerdb. This $R$ package is restricted to prediction of conserved miRNA targets only, which could explain why we do not obtain any correlations for some miRNAs and why the number of correlated targets is much higher in our tool for some miRNAs. In our tool we implemented the database for non-conserved miRNA targets as well and we also implemented DIANA-Tarbase and DIANA-microT-CDS in the miRNA target prediction step. For the comparison on protein expression levels (Table 10), a few pancreatic cancer-associated proteins were found in both tools. Interestingly, each of the tools also identified a unique set of true targets from the defined benchmarking set of KEGG pathway pancreatic cancer, suggesting that it might be of interest to use both tools for studying miRNA functions in pancreatic cancer. In addition to genes in KEGG pathway pancreatic 
cancer, there could of course be other relevant targets to study in a pancreatic cancer context.

The limitation of our pipeline is its current restriction to pancreatic cancer, as the correlations are based on data derived from pancreatic cancer tissue. To expand the pipeline for other cancer types, the pre-processing and inclusion of the miRNA, mRNA and protein expression levels is required for each cancer type. We are currently extending the pipeline to provide the same functionality for breast cancer miRNAs.

\section{Conclusions}

The developed pipeline is proven useful for generating shortlist of relevant target genes for 15 miRNAs that are differentially expressed in PDAC, along with their enriched GO terms, KEGG pathways, and significant correlations. Predicted miRNA-mRNA interactions in conjunction with correlation analyses of expression levels provides support for miRNA-mediated regulation. The pipeline is applicable to any mature miRNA in the context of pancreatic cancer. In the future, this pipeline could be further developed to include other cancer types by incorporating the corresponding miRNA, mRNA and protein expression levels of other TCGA cancer types. Our results and previously published data suggest that hsa-miR-885-5p could act as a tumor suppressor and should be experimentally validated in PDAC.

\section{Methods}

\section{Data}

\section{Differentially expressed microRNAs}

A published dataset of 15 significantly altered miRNAs detected in plasma of PDAC patients at the time of diagnosis were used in this study (Table 11) [7]. These circulating miRNAs have been identified in plasma samples from patients diagnosed with PDAC and admitted for surgery at the Department of Surgery, Umeå university hospital. MiRNA isolates from 23 PDAC patients and 22 controls were analyzed by RT-qPCR for 372 validated miRNAs using Human Panel I (V.4, Exiqon, Vedbaek, Denmark). The combination of these 15 miRNAs generated an AUC of 0.96 compared to 0.92 for CA 19-9 at time of diagnosis.

\section{Expression data}

MiRNA and mRNA expression data have previously been generated by next-generation sequencing (seq) within TCGA Research Network (http://cancergenome.nih.gov/). The miRNA-seq isoform expression quantification data files available at the GDC portal (https://portal.gdc.cancer. gov/) from pancreatic adenocarcinoma (TCGA-PAAD) samples were used. For mRNA expression data, $\log 2$ (fpkm +1 ) values for 183 samples were downloaded from xena browser (https://xenabrowser.net/datapages/). TCGAPAAD expression data were derived from 1 epithelial
Table 11 Significantly altered plasma miRNAs in pancreatic cancer patients [7]. Regulation describes whether the miRNA was found up- or down-regulated in pancreatic cancer patients

\begin{tabular}{lll}
\hline miRNA & Regulation & $F C$ \\
\hline hsa-miR-144-3p & Down & 0.4 \\
hsa-miR-106b-5p & Down & 0.8 \\
hsa-miR-451a & Down & 0.5 \\
hsa-miR-101-3p & Down & 0.7 \\
hsa-miR-26a-5p & Down & 0.6 \\
hsa-miR-574-3p & Up & 1.5 \\
hsa-miR-885-5p & Up & 3.9 \\
hsa-miR-130b-3p & Up & 1.5 \\
hsa-miR-34a-5p & Up & 2.2 \\
hsa-miR-24-3p & Up & 1.2 \\
hsa-miR-22-5p & Up & 1.4 \\
hsa-let-7d-3p & Up & 1.3 \\
hsa-miR-197-3p & $U p$ & 1.4 \\
hsa-miR-423-3p & $U p$ & 1.3 \\
hsa-miR-122-5p & $U p$ & 2.5 \\
\hline
\end{tabular}

FC fold change

neoplasm, 5 cystic, mucinous or serous neoplasms, 31 adenomas or adenocarcinomas and 146 ductal or lobular neoplasms. The R package org. Hs.eg.db was used for converting Ensembl-IDs to HUGO Gene Nomenclature Committee (HGNC) symbols [50].

Protein expression levels of 218 proteins, analyzed by RPPA, on tissue samples provided by TCGA were obtained from 98 TCGA-PAAD samples [24]. Level 4 protein expression data was downloaded from TCPA (http:// bioinformatics.mdanderson.org/main/TCPA:Overview) by accessing the data portal (http://tcpaportal.org/tcpa/ download.html). TCPA expression data were derived from 2 cystic, mucinous or serous neoplasms, 12 adenomas or adenocarcinomas and 84 ductal or lobular neoplasms. The protein names were modified to gene names by using the information from TCPA 'My Protein' resource.

\section{Annotation of mature miRNAs}

MiRNA sequencing expression data found in TCGA does not contain information about $-3 p$ or $-5 p$ arm. This problem has previously been addressed by Kuo et al. (2015) that developed a Python script to interrogate this information. Their idea was applied here using $\mathrm{R}$. The miRNA isoform expression quantification data was utilized from TCGA. A reads per million (rpm) threshold of 1 for calling a gene expressed was applied [51, 52]. Kuo et al. [53] only included the top three expressions of each isoforms for each mature miRNA, whereas here, all values with $\mathrm{rpm} \geq 1$ were summarized for each miRNA. A 'gdc sample sheet', containing information such as file ID and sample ID for each PAAD sample, 
was also downloaded. The gdc sample sheet was used as input for the $\mathrm{R}$ function. For each quantification file, the reads per million (rpm) counts $\geq 1$ were summarized for each MIMA-ID using the plyr package [54]. All samples were merged into one table using merge() function, with option 'all' $=\mathrm{T}$, resulting in samples as colnames and MIMAT-IDs in the first column. After merging, data was changed to $\log 2(\mathrm{rpm}+1)$ and hence all 'NA' values were changed to 0 . The MIMAT-ID is an identity for each unique miRNA. A file containing information on MIMAT-ID and mature miRNA names (hsa.gff3) was downloaded from miRBase version 22 (www.mirbase. org). The MIMAT-IDs were translated using Perl version 5.18.2 and the miRNA nomenclature file (hsa.gff3). The final output was a table containing the expression levels for each mature miRNA and PAAD sample.

\section{Bioinformatics pipeline}

The pipeline was built in $\mathrm{R}$ version 3.5 .1 [55] and consists of miRNA target prediction, correlation analyses and functional enrichment analysis (Fig. 1). The $\mathrm{R}$ script, along with instructions for installing and running the pipeline can be accessed through https:// emmbor.shinyapps.io/mirfa/.

\section{miRNA target prediction}

In the implemented pipeline, we included following databases: one experimentally validated database; DIANATarBase v7 [25] and two in silico target prediction databases; DIANA-microT-CDS [26] and TargetScan v7.1 [27] for miRNA target prediction. A prediction score threshold was set to 0.7 for DIANA-microT-CDS. DIANA-TarBase v7 (http://diana.imis.athena-innovation.gr/DianaTools/index. php?r=tarbase/index) and DIANA-microT-CDS (http:// diana.imis.athena-innovation.gr/DianaTools/index.php) were downloaded. In addition, three different TargetScan v7.1 databases were downloaded; predicted targets for conserved miRNA families, predicted conserved sites for miRNAs and predicted non-conserved sites miRNAs (http:// www.targetscan.org/vert_71/). The overlap of identified miRNA target genes from Tarbase, microT-CDS and TargetScan was visualized with Venn diagrams, generated by the VennDiagram package [56].

The downloaded miRNA target prediction databases, along with miRNA, mRNA, and protein expression levels were combined into an sqlite database using sqlite3, called 'mirna_database.sqlite' available at https://1drv.ms/u/ s!Ap_ICu6nvktNgW6Y68Zkp1HTx0vE, and was queried from the pipeline using RSQLite package [57].

\section{Correlation analysis}

Correlation analyses were performed to increase the reliability of the predicted miRNA targets by correlating miRNA expression levels to mRNA and protein expression levels of its target genes. Correlations between expression levels of miRNA and mRNA, and miRNA and protein expression levels were performed. The cor() and cor.test() functions were applied using the Pearson's correlation method. A significance threshold was set at $\alpha=0.05$ and multiple testing adjustment using the Benjamini-Hochberg method was performed. Since miRNAs can function as up- or down-regulators of mRNAs, positive and negative correlations were included in subsequent analyses $[2,3]$.

\section{Functional enrichment}

Functional enrichment analysis was performed on the correlated miRNA targets [18]. Functional enrichment included enrichment analyses of GO terms and KEGG pathways. The goana() and kegga() functions from the edgeR package were implemented [58, 59]. Criteria of at least 5 genes significantly enriched for a given term and a false discovery rate of 0.05 were used as cutoffs.

\section{Shiny web app}

To increase the data availability and make miRFA useful for wider community, the pipeline was run for all microRNAs in the TCGA-PAAD dataset (775 miRNAs). The results can be obtained through a shiny application at https://emmbor. shinyapps.io/mirfa/ [17]. Download of data is available at the bottom of each table and Venn diagram plot.

\section{Survival analysis}

Kaplan-Meier curves were generated using $\mathrm{R}$ packages survminer, RTCGA.clinical and survival packages with median as cut-off [60-63]. P-values were adjusted for multiple testing using the Benjamini-Hochberg method.

\section{Network analysis}

Hub genes were identified separately for positively and negatively correlated targets for hsa-miR-885-5p. Positively or negatively correlated gene lists were submitted to the STRING database version 10.5 (https://string-db.org/ cgi/input.pl) with Homo sapiens as organism and a high confidence' interaction score (0.7) [64]. The cytohubba plugin [65] was used in Cytoscape version 3.7.0 to show the ranked, top 10 hub genes. ClueGO version 2.5.2 together with CluePedia version 1.5.2 were used to see the overlapping genes between KEGG pathways for positive and negatively correlated targets for hsa-miR-885-5p. Only correlated targets with a Pearson's correlation coefficient $\geq 0.4$ or $\leq-0.4$ were included in the analysis.

\section{Additional files}

Additional file 1: Hsa-miR-22-5p KEGG pathways. Table of enriched KEGG pathways for hsa-miR-22-5p. ' $N$ ' describes number of genes 
described for a specific KEGG pathway, 'DE' describes number of genes enriched for the pathway and 'P.DE' is the $p$-value. (CSV $5 \mathrm{~kb}$ )

Additional file 2: Hsa-miR-24-3p KEGG pathways. Table of enriched KEGG pathways for hsa-miR-24-3p. ' $N$ ' describes number of genes described for a specific KEGG pathway, 'DE' describes number of genes enriched for the pathway and 'P.DE' is the p-value. (CSV 3 kb)

Additional file 3: Hsa-miR-106b-5p KEGG pathways. Table of enriched KEGG pathways for hsa-miR-106b-5p. ' $N$ ' describes number of genes described for a specific KEGG pathway, 'DE' describes number of genes enriched for the pathway and 'P.DE' is the p-value. (CSV $3 \mathrm{~kb}$ )

Additional file 4: Hsa-miR-885-5p KEGG pathways. Table of enriched KEGG pathways for hsa-miR-885-5p. ' $N$ ' describes number of genes described for a specific KEGG pathway, 'DE' describes number of genes enriched for the pathway and 'P.DE' is the p-value. (CSV 3 kb)

Additional file 5: Significant correlations on mRNA expression level. This table contains all significant correlations on mRNA expression level for all 15 miRNAs. 'Gene' is the predicted miRNA target, ' $P C C$ ' = Pearson's correlation coefficient, 'adj_P_value' = adjusted p-value. (CSV 880 kb)

Additional file 6: Figure S1. Venn diagram of DIANA-TarBase v7, DIANA-microT-CDS and TargetScan v7.1 for each miRNA. The R package VennDiagram was used to generate the overlap of identified miRNA target genes. DIANA-TarBase $v 7=$ Tarbase (pink), DIANA-microT-CDS $=$ microT-CDS (blue), TargetScan v.7.1 = TargetScan (grey). Figure S2. Overall survival analysis for each miRNA. Kaplan-Meier curves were generated with median as cut-off. Expression $=0$ is the group that has a value below median and expression=1 is the group that has a value above median. $\mathrm{P}$ values are displayed before multiple hypothesis correction, after multiple hypothesis correction with Benjamini-Hochberg, no miRNA was significant. (DOCX $1105 \mathrm{~kb})$

\section{Abbreviations}

AUC: Area under curve; CA 19-9: Carbohydrate antigen 19-9; DIANA: DNA intelligent analysis; GO: Gene ontology; HGNC: HUGO gene nomenclature committee; hsa: Homo sapiens; KEGG: Kyoto encyclopedia of genes and genomes; miRFA: miRNA functional analysis; miRNA: microRNA; miRNP: miRNA ribonucleoprotein complex: PAAD: Pancreatic adenocarcinoma; PCC: Pearsons's correlation coefficient; PDAC: Pancreatic ductal adenocarcinoma; rpm: Reads per million; RPPA: Reverse-phase protein arrays; seq: Sequencing; TCGA: The cancer genome atlas; TCPA: The cancer proteome atlas; TNM: tumor-node-metastasis

\section{Acknowledgements}

The authors thank Angelica Lindlöf, Björn Olsson, Ola Billing, Oskar Franklin and Hanna Nyström for great input and valuable discussions. We also thank Tejaswi Venkata Satya Badam and Thomas Hillerton for programming assistance. We also acknowledge James Mason for English proofreading.

\section{Authors' contributions}

EB and HAW developed the pipeline, and shiny application. EB performed the analyses and wrote the manuscript. ZL and MS supervised the project and revised the manuscript. All authors have read and approved the final manuscript.

\section{Funding}

This study was funded by grants from the Swedish Research Council (no 2016-02990), the Swedish Cancer Society (no CAN 2016/643) and Västerbotten County Council (no VLL-583411 and VLL-549731). The funding bodies have not had any roles in the design of the study, and collection, analysis and interpretation of data or in writing the manuscript.

\section{Availability of data and materials}

The code for miRFA is available at https://github.com/emmbor/miRFA and our database 'mirna_database.sqlite' can be downloaded from https://1 drv. ms/u/s!Ap_ICu6nvktNgW6Y68Zkp1HTxOvE. The datasets supporting the conclusions of this article are included within the article and its additional files. Results of all miRNAs detected in TCGA-PAAD can be obtained through our shiny application at https://emmbor.shinyapps.io/mirfa/.

\section{Ethics approval and consent to participate}

All data used in the study is publicly available, hence no ethical approval was needed.

\section{Consent for publication}

Not applicable.

\section{Competing interests}

The authors declare that they have no competing interests.

\section{Author details}

'Department of Surgical and Perioperative Sciences, Umeå University, Umeå, Sweden. ${ }^{2}$ School of bioscience, Systems Biology Research Centre, University of Skövde, Skövde, Sweden. ${ }^{3}$ Department of Physics, Chemistry and Biology, Bioinformatics, Linköping University, Linköping, Sweden.

Received: 14 December 2018 Accepted: 28 June 2019

Published online: 16 July 2019

\section{References}

1. Bhaskaran M, Mohan M. MicroRNAs: history, biogenesis, and their evolving role in animal development and disease. Vet Pathol. 2014;51(4):759-74.

2. Vasudevan S, Tong Y, Steitz JA. Switching from repression to activation: microRNAs can up-regulate translation. Science. 2007;318(5858):1931-4.

3. Rusk N. When microRNAs activate translation. Nat Methods. 2008;5(2):122-3.

4. Wilczynska A, Bushell M. The complexity of miRNA-mediated repression. Cell Death Differ. 2015;22(1):22-33.

5. Orang AV, Safaralizadeh R, Kazemzadeh-Bavili M. Mechanisms of miRNAmediated gene regulation from common downregulation to mRNA-specific upregulation. Int J Genomics. 2014;970607.

6. Pelosi E, Castelli G, Testa U. Pancreatic Cancer: molecular characterization, clonal evolution and Cancer stem cells. Biomedicines. 2017;5(4):65.

7. Franklin O, Jonsson P, Billing O, Lundberg E, Öhlund D, Nyström H, et al. Plasma micro-RNA alterations appear late in pancreatic Cancer. Ann Surg. 2018;267(4): 775-81.

8. Poruk KE, Gay DZ, Brown K, Mulvihill JD, Boucher KM, Scaife CL, et al. The clinical utility of CA 19-9 in pancreatic adenocarcinoma: diagnostic and prognostic updates. Curr Mol Med. 2013;13(3):340-51.

9. Schultz NA, Dehlendorff C, Jensen BV, Bjerregaard JK, Nielsen KR, Bojesen SE, et al. MicroRNA biomarkers in whole blood for detection of pancreatic cancer. JAMA. 2014;311(4):392-404.

10. Xu J, Cao Z, Liu W, You L, Zhou L, Wang C, et al. Plasma miRNAs effectively distinguish patients with pancreatic Cancer from controls: a multicenter study. Ann Surg. 2016;263(6):1173-9.

11. Liu B, Li J, Cairns MJ. Identifying miRNAs, targets and functions. Brief Bioinform. 2014;15(1):1-19.

12. Ru Y, Kechris KJ, Tabakoff B, Hoffman P, Radcliffe RA, Bowler R, et al. The multiMiR R package and database: integration of microRNA-target interactions along with their disease and drug associations. Nucleic Acids Res. 2014;42(17):e133.

13. Zhang J, Storey KB. RBiomirGS: an all-in-one miRNA gene set analysis solution featuring target mRNA mapping and expression profile integration. PeerJ. 2018;6:e4262.

14. Vila-Casadesús M, Gironella M, Lozano JJ. MiRComb: an R package to analyse miRNA-mRNA interactions. Examples across five digestive cancers. PLoS One. 2016;11(3):e0151127.

15. Le TD, Zhang J, Liu L, Liu H, Li J. miRLAB: an R based dry lab for exploring miRNA-mRNA regulatory relationships. PLoS One. 2015;10(12):e0145386.

16. Ahmed M, Nguyen $H$, Lai T, Kim DR. miRCancerdb: a database for correlation analysis between microRNA and gene expression in cancer. BMC Res Notes. 2018;11(1):103.

17. Chang W, Cheng J, Allaire JJ, Xie Y, McPherson J. Shiny: Web Application Framework for R: 2018.

18. Vlachos IS, Zagganas K, Paraskevopoulou MD, Georgakilas G, Karagkouni D, Vergoulis T, et al. DIANA-miRPath v3.0: deciphering microRNA function with experimental support. Nucleic Acids Res. 2015;43(W1):W460-6.

19. Pinzon N, Li B, Martinez L, Sergeeva A, Presumey J, Apparailly F, et al. microRNA target prediction programs predict many false positives. Genome Res. 2017;27(2):234-45.

20. Lee YJD, Kim V, Muth DC, Witwer KW. Validated MicroRNA Target Databases: An Evaluation. Drug Dev Res. 2015;76(7):389-96. 
21. Seo J, Jin D, Choi $\mathrm{C}-\mathrm{H}$, Lee $\mathrm{H}$. Integration of MicroRNA, mRNA, and protein expression data for the identification of Cancer-related MicroRNAs. PLoS One. 2017;12(1):e0168412.

22. Bong IPN, Ng CC, Baharuddin P, Zakaria Z. MicroRNA expression patterns and target prediction in multiple myeloma development and malignancy. Genes Genomics. 2017;39(5):533-40.

23. Zhang $W$, Zang J, Jing $X$, Sun Z, Yan W, Yang D, et al. Identification of candidate miRNA biomarkers from miRNA regulatory network with application to prostate cancer. J Transl Med. 2014;12:66

24. Li J, Lu Y, Akbani R, Ju Z, Roebuck PL, Liu W, et al. TCPA: a resource for cancer functional proteomics data. Nat Methods. 2013;10(11):1046-7.

25. Vlachos IS, Paraskevopoulou MD, Karagkouni D, Georgakilas G, Vergoulis T, Kanellos I, et al. DIANA-TarBase v7.0: indexing more than half a million experimentally supported miRNA:mRNA interactions. Nucleic Acids Res. 2015:43(Database issue):D153-9.

26. Reczko M, Maragkakis M, Alexiou P, Grosse I, Hatzigeorgiou AG. Functional microRNA targets in protein coding sequences. Bioinformatics (Oxford, England). 2012;28(6):771-6.

27. Agarwal V, Bell GW, Nam J-W, Bartel DP. Predicting effective microRNA target sites in mammalian mRNAs. eLife. 2015;4:101.

28. Backes $C$, Khaleeq QT, Meese E, Keller A. miEAA: microRNA enrichment analysis and annotation. Nucleic Acids Res. 2016;44(W1):W110-6.

29. Lu M, Shi B, Wang J, Cao Q, Cui Q. TAM: a method for enrichment and depletion analysis of a microRNA category in a list of microRNAs. BMC Bioinformatics. 2010;11:419.

30. Stockel D, Kehl T, Trampert P, Schneider L, Backes C, Ludwig N, et al. Multi-omics enrichment analysis using the GeneTrail2 web service. Bioinformatics. 2016;32(10):1502-8.

31. Hazra A, Gogtay N. Biostatistics series module 6: correlation and linear regression. Indian J Dermatol. 2016;61(6):593-601.

32. Zhang Z, Yin J, Yang J, Shen W, Zhang C, Mou W, et al. miR-885-5p suppresses hepatocellular carcinoma metastasis and inhibits Wnt/betacatenin signaling pathway. Oncotarget. 2016;7(46):75038-51.

33. Indersie E, Lesjean S, Hooks KB, Sagliocco F, Ernault T, Cairo S, et al. MicroRNA therapy inhibits hepatoblastoma growth in vivo by targeting beta-catenin and Wnt signaling. Hepatol Commun. 2017:1(2):168-83.

34. Vychytilova-Faltejskova P, Pesta M, Radova L, Liska V, Daum O, Kala Z, et al. Genome-wide microRNA expression profiling in primary tumors and matched liver metastasis of patients with colorectal Cancer. Cancer Genomics Proteomics. 2016;13(4):311-6.

35. Ganepola GA, Rutledge JR, Suman P, Yiengpruksawan A, Chang DH. Novel blood-based microRNA biomarker panel for early diagnosis of pancreatic cancer. World J Gastrointest Oncol. 2014;6(1):22-33.

36. Hussein NA, Kholy ZA, Anwar MM, Ahmad MA, Ahmad SM. Plasma miR-22 $3 p$, miR-642b-3p and miR-885-5p as diagnostic biomarkers for pancreatic cancer. J Cancer Res Clin Oncol. 2017;143(1):83-93.

37. Wordeman L, Mitchison TJ. Identification and partial characterization of mitotic centromere-associated kinesin, a kinesin-related protein that associates with centromeres during mitosis. J Cell Biol. 1995:128(1-2):95-104.

38. Cordeiro MH, Smith RJ, Saurin AT. A fine balancing act: a delicate kinasephosphatase equilibrium that protects against chromosomal instability and cancer. Int J Biochem Cell Biol. 2018;96:148-56.

39. Hussain M, Lu Y, Liu YQ, Su K, Zhang J, Liu J, et al. Skp1: implications in cancer and SCF-oriented anti-cancer drug discovery. Pharmacol Res. 2016;111:34-42.

40. Dong M, Nio Y, Guo KJ, Tamura K, Tian YL, Dong YT. Epidermal growth factor and its receptor as prognostic indicators in Chinese patients with pancreatic cancer. Anticancer Res. 1998;18(6b):4613-9.

41. Luo GP, Long J, Qiu LX, Liu C, Xu J, Yu XJ. Role of epidermal growth factor receptor expression on patient Survival in pancreatic Cancer: a metaanalysis. Pancreatology. 2011;11(6):595-600.

42. Park SJ, Gu MJ, Lee DS, Yun SS, Kim HJ, Choi JH. EGFR expression in pancreatic intraepithelial neoplasia and ductal adenocarcinoma. Int J Clin Exp Pathol. 2015;8(7):8298-304.

43. Tsiambas E, Karameris A, Lazaris AC, Talieri M, Triantafillidis JK, Cheracakis $P$, et al. EGFR alterations in pancreatic ductal adenocarcinoma: a chromogenic in situ hybridization analysis based on tissue microarrays. HepatoGastroenterol. 2006:53(69):452-7.

44. Ueda S, Ogata S, Tsuda H, Kawarabayashi N, Kimura M, Sugiura Y, et al. The correlation between cytoplasmic overexpression of epidermal growth factor receptor and tumor aggressiveness - poor prognosis in patients with pancreatic ductal adenocarcinoma. Pancreas. 2004;29(1):E1-8.
45. Valsecchi ME, McDonald M, Brody JR, Hyslop T, Freydin B, Yeo CJ, et al. Epidermal growth factor receptor and insulinlike growth factor 1 receptor expression predict poor survival in pancreatic ductal adenocarcinoma. Cancer-Am Cancer Soc. 2012;118(14):3484-93.

46. Saukkonen K, Hagstrom J, Mustonen H, Juuti A, Nordling S, Kallio P, et al. PROX1 and beta-catenin are prognostic markers in pancreatic ductal adenocarcinoma. BMC Cancer. 2016;16:472.

47. Martinez-Useros J, Li W, Georgiev-Hristov T, Fernandez-Acenero MJ, BorreroPalacios A, Perez N, et al. Clinical implications of NRAS overexpression in Resectable pancreatic adenocarcinoma patients. Pathol Oncol Res. 2017; 12(8):e0182044.

48. Wu W-S, Tu B-W, Chen T-T, Hou S-W, Tseng JT. CSmiRTar: condition-specific microRNA targets database. PLoS One. 2017;12(7):e0181231.

49. Csardi G. targetscan. Hs.Eg.Db: TargetScan miRNA target predictions for human; 2013.

50. Carlson M. org.Hs.eg.db: Genome wide annotation for Human; 2017.

51. Hebenstreit D, Fang M, Gu M, Charoensawan V, van Oudenaarden A, Teichmann SA. RNA sequencing reveals two major classes of gene expression levels in metazoan cells. Mol Syst Biol. 2011;7:497.

52. Wagner GP, Kin K, Lynch VJ. A model based criterion for gene expression calls using RNA-seq data. Theory Biosci. 2013;132(3):159-64.

53. Kuo WT, Su MW, Lee $Y L$, Chen $\mathrm{CH}$, Wu CW, Fang WL, et al. Bioinformatic interrogation of $5 p$-arm and 3p-arm specific miRNA expression using TCGA datasets. J Clin Med. 2015:4(9):1798-814.

54. Wickham $H$. The Split-apply-combine strategy for data analysis. J Stat Softw. 2011:40(1):1-29. http://www.jstatsoft.org/v40/i01/.

55. R core team. R: A Language and Environment for Statistical Computing Vienna, Austria2017 [Available from: https://www.R-project.org/.

56. Chen H. VennDiagram: Generate high-resolution Venn and Euler plots 2018. Available from: https://CRAN.R-project.org/package=VennDiagram.

57. Müller K, Wickham H, James DA, Falcon S. [ctb] SA, Healy Lc. RSQLite: 'SQLite' Interface for R; 2018.

58. McCarthy DJ, Chen Y, Smyth GK. Differential expression analysis of multifactor RNA-Seq experiments with respect to biological variation. Nucleic Acids Res. 2012;40(10):4288-97.

59. Robinson MD, McCarthy DJ, Smyth GK. edgeR: a Bioconductor package for differential expression analysis of digital gene expression data. Bioinformatics. 2010;26(1):139-40.

60. Kassambara AK, survminer M. Drawing Survival curves using 'ggplot2; 2018.

61. Kosinski M. RTCGA.Clinical: clinical datasets from the Cancer genome atlas project; 2018.

62. Therneau T. A Package for Survival Analysis in S; 2015.

63. Therneau TG, Modeling Survival P. Data: extending the $\{C\} 0 x$ model. New York: Springer; 2000.

64. Szklarczyk D, Franceschini A, Wyder S, Forslund K, Heller D, Huerta-Cepas J, et al. STRING v10: protein-protein interaction networks, integrated over the tree of life. Nucleic Acids Res. 2015;43(Database issue):D447-52.

65. Chin $\mathrm{CH}$, Chen $\mathrm{SH}, \mathrm{Wu} \mathrm{HH}, \mathrm{Ho} \mathrm{CW}$, Ko MT, Lin CY. cytoHubba: identifying hub objects and sub-networks from complex interactome. BMC Syst Biol. 2014;8(Suppl 4):S11.

\section{Publisher's Note}

Springer Nature remains neutral with regard to jurisdictional claims in published maps and institutional affiliations.

Ready to submit your research? Choose BMC and benefit from:

- fast, convenient online submission

- thorough peer review by experienced researchers in your field

- rapid publication on acceptance

- support for research data, including large and complex data types

- gold Open Access which fosters wider collaboration and increased citations

- maximum visibility for your research: over $100 \mathrm{M}$ website views per year

At BMC, research is always in progress.

Learn more biomedcentral.com/submission 